Check for updates

Cite this: RSC Adv., 2018, 8, 25592

Received 23rd May 2018

Accepted 7th July 2018

DOI: $10.1039 / \mathrm{c} 8 \mathrm{ra04380a}$

rsc.li/rsc-advances

\title{
Chitosan capped nickel oxide nanoparticles as a saturable absorber in a tunable passively Q- switched erbium doped fiber laser
}

\author{
Harith Ahmad, iD $\dagger^{*}$ Siti Aisyah Reduan and Norazriena Yusoff
}

\begin{abstract}
Nickel oxide ( $\mathrm{NiO}$ ) nanoparticles successfully prepared from a nickel(॥) chloride hexahydrate precursor are used to form a chitosan capped $\mathrm{NiO}$ nanoparticle thin film to serve as a saturable absorber (SA) for the generation of passively Q-switched pulses in an erbium doped fiber laser (EDFL). The NiO/chitosan SA based EDFL is able to generate stable pulsed outputs at a low threshold pump power of $104.90 \mathrm{~mW}$ with a central wavelength at $1562 \mathrm{~nm}$. The highest pulse energy obtainable by the system is $15.30 \mathrm{~nJ}$ at a repetition rate of $42.66 \mathrm{kHz}$ and a pulse duration of $2.02 \mu \mathrm{s}$. The laser has a spectral range of $58 \mathrm{~nm}$ from 1522 to $1580 \mathrm{~nm}$, covering the $C$ and L bands and even portions of the $S$ band. This study experimentally demonstrates that the potential of the NiO/chitosan film as an SA material for $\mathrm{Q}$ switching operations, combined with the biocompatibility, non-toxicity and high thermal resistance of Chitosan, holds great prospects for a broad range of applications.
\end{abstract}

\section{Introduction}

Q-switched and mode-locked fiber lasers are the focus of significant research efforts due to their ability to generate high peak powered outputs, in the range of tens of kilowatts to megawatts while at the same time retaining a compact and rugged form factor that is relatively easy and cost-effective to fabricate. ${ }^{1,2}$ Q-switched fiber lasers in particular are preferred for most practical applications, as even though the pulsed output generated is slower than that of mode-locked lasers, Qswitched lasers are still capable of generating pulses with significant peak powers. ${ }^{3,4}$ Additionally, Q-switched fiber lasers also do not require delicate adjustment and balancing of the dispersion and nonlinearity within the laser cavity as is usually the case with mode-locked fiber lasers, thus giving them an added advantage. ${ }^{5}$ These highly desirable traits have thus seen the integration of Q-switched fiber lasers into a multitude of real world applications such as range finding, ${ }^{6}$ medicine, ${ }^{7}$ micromachining, ${ }^{8}$ fiber optic sensing ${ }^{9}$ and telecommunications. ${ }^{\mathbf{1 0}}$

Q-switched pulses are typically generated through the use of a saturable absorber (SA), which modulates a continuous wave (CW) output into a periodic pulse train. For a long time, semiconductor saturable absorber mirrors (SESAMs) were the preferred choice of SA for generating Q-switched pulses in laser systems including fiber lasers and solid state lasers. However,

Photonics Research Center, University of Malaya, 50603 Kuala Lumpur, Malaysia. E-mail: harith@um.edu.my

$\uparrow$ Visiting Professor at the Department of Physics, Faculty of Science and Technology, Airlangga University, Surabaya 60115, Indonesia.
SESAMs were bulky, fragile and expensive to fabricate, thus limiting their use to only applications in which they would be necessary. However, advances in materials science and fabrication techniques have now seen a new generation of SAs coming to the forefront. These new SAs, made from 2-dimensional (2D) and 3-dimensional (3D) materials such as carbon nanotubes (CNTs), ${ }^{11}$ graphene, ${ }^{12}$ topological insulators (TIs), ${ }^{13}$ transition metal dichalcogenides (TMDs), ${ }^{14}$ and black phosphorus ${ }^{\mathbf{1 5 , 1 6}}$ have seen increasing use in various $\mathrm{Q}$-switched fiber laser systems. SAs have also been used in solid state laser systems, as demonstrated by B. Xu et al., who fabricated an SA based on a few $\mathrm{Bi}_{2} \mathrm{Se}_{3}$ and $\mathrm{MoS}_{2}$ layers to generate Q-switched pulses in solid state lasers using $\mathrm{Nd}^{-\mathrm{LiYF}_{4}}$ (YLF) and $\mathrm{Nd}: \mathrm{YAIO}_{3}$ crystals respectively. ${ }^{17,18}$

In this regard, transition metal oxide (TMO)-based SAs have now emerged as a new branch of SA capable materials ${ }^{19-21}$ with significant potential for inducing Q-switched pulses in fiber laser. This had been demonstrated by B. Xuekun et al. ${ }^{22}$ who used a ferro-oxide $\left(\mathrm{Fe}_{3} \mathrm{O}_{4}\right)$ nanoparticle based SA for Qswitching operation in an erbium doped fiber laser (EDFL) cavity. The proposed laser generated pulses with energy of 23.67 $\mathrm{nJ}$ and width of $3.20 \mu$ s at a repetition rate of $33.30 \mathrm{kHz}$ and peak power exceeding $110 \mathrm{~mW}$. Additionally, J. Guo et al. ${ }^{23}$ demonstrated a passively Q-switched EDFL capable of generating an output with a pulse width as narrow as $1.15 \mu$ s and pulse energy as high as of $21.19 \mathrm{~nJ}$ at a repetition rate of $81.28 \mathrm{kHz}$ using the SA fabricated from indium tin oxide (ITO) nanocrystals.

The aforementioned reports, along with other works, ${ }^{24-26}$ all indicate clearly the significant potential that TMOs based SAs have in the generation of pulsed outputs. Of the many TMOs explored for this application, nickel oxide (NiO) is seen to be 
a prime candidate for the development of SAs capable of generating Q-switched outputs. This is due primarily to the substantial electrical, mechanical and optical properties of NiO nanoparticles, which include a wide absorption band, low saturation intensity and fast response time. ${ }^{24,27} \mathrm{NiO}$ nanoparticles also have significantly advantageous nonlinear photonic properties such as a good modulation depth, high optical damage threshold and low saturation intensity. ${ }^{28}$ These characteristics, combined with the relatively cost-effective and easy fabrication process of TMOs as SAs as well as its high chemical and thermal stability and environmentally friendly constitution, ${ }^{29}$ make it suitable for a variety of real world applications, including its usage in dye-sensitized solar cells, ${ }^{30}$ super capacitors, ${ }^{31,32}$ fiber and batteries $^{33}$ and catalyst materials. $^{34}$

In this work, NiO nanoparticles are synthesized and mixed with a polymer matrix to form thin films that are then used as SAs for generating a tunable Q-switched laser output from a Cband EDFL cavity. The sonochemical method is used to fabricate the NiO nanoparticles followed by a simple solution processing technique with chitosan to produce a $\mathrm{NiO} /$ chitosan thin film. Chitosan is chosen as the polymer host for the NiO nanoparticles due to its excellent film-forming capacity as well as high biocompatibility, good bioactivity, high biodegradability and non-toxicity as well as being readily available in abundance. ${ }^{35,36}$ The $\mathrm{NiO} /$ chitosan $\mathrm{SA}$ is then integrated into a Cband EDFL for the passive generation of Q-switched pulses. The $\mathrm{NiO} /$ chitosan based SA is expected to demonstrate a number of significant advantages, including low threshold pump power, broad wavelength tunability and high pulse generation stability that will make if significantly useful for real world applications.

\section{Fabrication and characterization of nickel oxide/chitosan saturable absorber (SA)}

For the synthesis of the NiO/chitosan thin film based SA, the following chemicals and reagents are first obtained. Nickel(II) chloride hexahydrate $\left(\mathrm{NiCl}_{2} \cdot 6 \mathrm{H}_{2} \mathrm{O}\right)$ at purity of $\geq 97 \%$, acetic acid at purity $\geq 99.8 \%$, and sodium hydroxide $(\mathrm{NaOH})$ are purchased from Sigma-Aldrich, while a $25 \%$ ammonia solution $\left(\mathrm{NH}_{3} \cdot \mathrm{H}_{2} \mathrm{O}\right)$ is obtained from $\mathrm{R} \& \mathrm{M}$ Chemicals. High viscosity chitosan flakes is obtained from Fluka BioChemika. All reagents are of analytical grade and used as received without further purification.

The $\mathrm{NiO} /$ chitosan film is synthesized by first preparing $1 \mathrm{M}$ of Ni precursor by dissolving an appropriate amount of $\mathrm{NiCl}_{2}-$ $\cdot 6 \mathrm{H}_{2} \mathrm{O}$ powder in $20 \mathrm{~mL}$ of distilled (DI) water while being stirred at room temperature. A $13 \mathrm{~mL}$ aqueous solution of $\mathrm{NaOH}$ $(1 \mathrm{M})$ that functions as a structure-directing agent is added to the solution while the stirring continues. $\mathrm{An} \mathrm{NH}_{3} \cdot \mathrm{H}_{2} \mathrm{O}$ solution is then added drop-wise to maintain the $\mathrm{pH}$ of the solution at a value of approximately 10 . Subsequently, the mixture is sonicated using probe type sonicator at 100 watt for 2 hours and the sonicated dispersions then centrifuged at $4000 \mathrm{rpm}$ for 20 min to obtain black precipitates which are washed with DI water and ethanol several times to eliminate residual impurities. The precipitates are then dried at $60{ }^{\circ} \mathrm{C}$ in an oven for 24 hours to remove residual ethanol. To ensure that any remaining hydroxide forms of $\mathrm{Ni}$ are reduced to $\mathrm{NiO}$, the black precipitate powder is annealed at $450{ }^{\circ} \mathrm{C}$ for 1 hour under atmospheric pressure.

The NiO/chitosan thin film is prepared by dispersing $50 \mathrm{mg}$ $\mathrm{NiO}$ powder homogeneously in $10 \mathrm{~mL}$ of DI water using ultrasonication before being mixed with $10 \mathrm{~mL}$ of chitosan solution at the same concentration and being stirred at a temperature of $70{ }^{\circ} \mathrm{C}$ for a period of 1 hour. The chitosan solution itself is prepared by dissolving high viscosity chitosan flakes in $1 \%$ acetic acid while under constant stirring at room temperature for 5 hours to ensure the chitosan powder to be completely dissolved. The obtained slurry is poured into a Petri dish and dried in the oven at $60^{\circ} \mathrm{C}$ until the desired $\mathrm{NiO} /$ chitosan film is formed. Fig. 1 shows the various key steps taken to form the $\mathrm{NiO} /$ chitosan thin film.

$\mathrm{NiO} /$ chitosan SA itself is fabricated by sandwiching a very small piece of the $\mathrm{NiO} /$ chitosan film between two single mode fiber ferrules using a fiber adaptor. A small amount of index matching gel is applied to the film and fiber ferrules to serve as an adhesive and at the same time used to minimize possible losses due to Fresnel reflection.

The crystalline phase of the nanoparticles is analyzed by a PANalytical Empyrean X-ray diffractometer (XRD) with monochromatized $\mathrm{Cu} \mathrm{K} \alpha$ radiation and $\lambda=1.5418 \AA$. The system is operating at a scanning rate of $0.02^{\circ}$ per second to over a $2 \theta$ range of $30^{\circ}$ to $80^{\circ}$. Fourier transform infrared (FTIR) spectra analysis of the thin film is carried out using a Perkin Elmer FTIRSpectrum 400 spectrometer at room temperature. A Field Emission Scanning Electron Microscope (FESEM) image is obtained by a Hitachi SU8220 operated at $1.0 \mathrm{kV}$ while energy dispersive Xray spectroscopy (EDX) analysis was carried out in conjunction with the FESEM analysis to confirm the material composition of

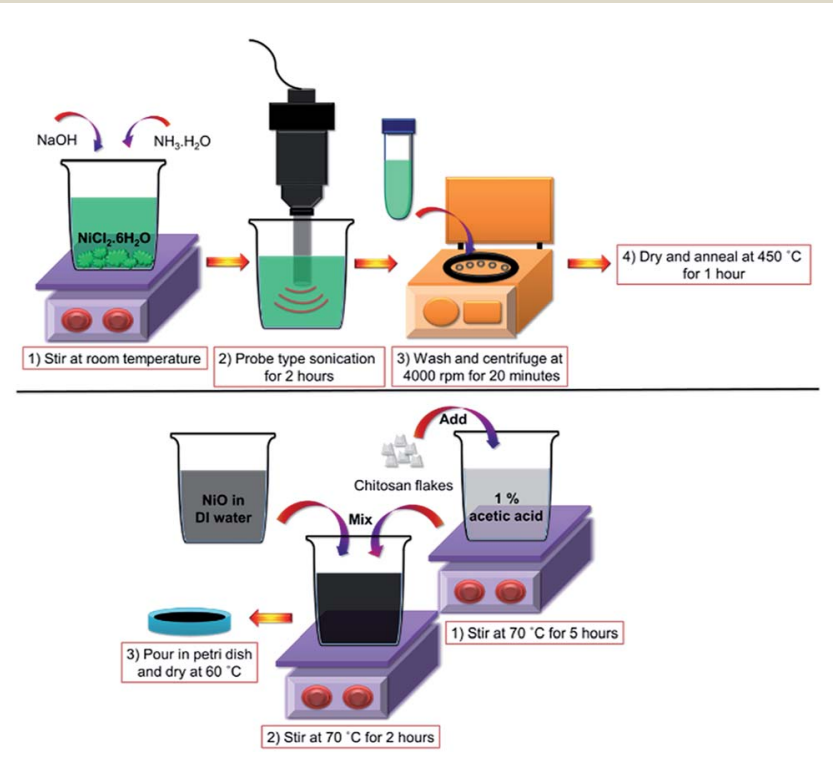

Fig. 1 Schematic representation for: (top) the preparation of $\mathrm{NiO}$ nanoparticles using the sonochemical method and (above) the fabrication of the $\mathrm{NiO} /$ chitosan thin film. 


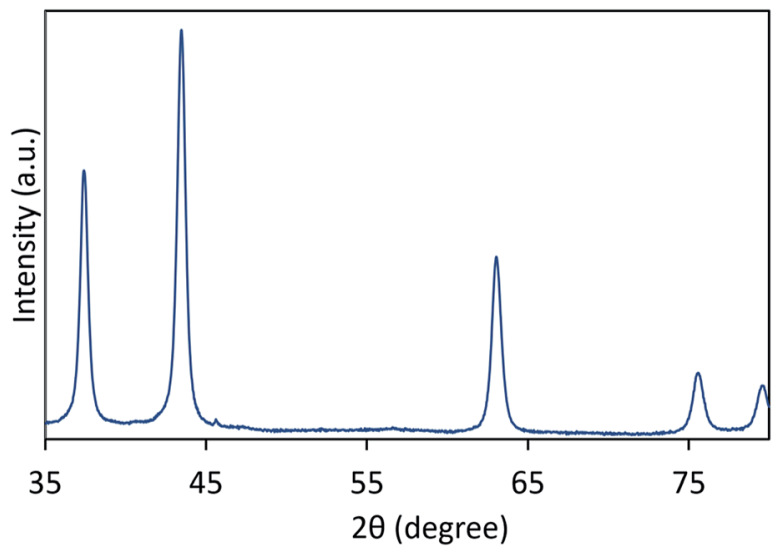

Fig. 2 The XRD pattern of $\mathrm{NiO}$ nanoparticles.

the SA. Fig. 2 shows the XRD pattern for the NiO nanoparticles synthesized using the sonochemical method. Diffraction peaks are observed at $2 \theta$ values of $31.9^{\circ}, 43.5^{\circ}, 63.2^{\circ}, 75.4^{\circ}$ and $79.4^{\circ}$, and are assigned to the (1 111$),\left(\begin{array}{lll}2 & 0 & 0\end{array}\right),\left(\begin{array}{lll}2 & 2 & 0\end{array}\right),\left(\begin{array}{lll}3 & 1 & 1\end{array}\right)$ and ( $\left(\begin{array}{lll}2 & 2 & 2\end{array}\right)$ planes of NiO. These XRD peaks of $\mathrm{NiO}$ are consistent with the data of the JCPDS 47-1049 file, reflecting the formation of NiO after the reaction process. ${ }^{37}$ The sharp peaks indicate the high crystallinity of the NiO nanoparticles and no other peaks are observed, indicating there are no impurities present and that the sample has high purity. The crystallite size of the NiO nanoparticles is determined to be $\sim 20 \mathrm{~nm}$ as calculated using the Scherrer equation with a full-width at half maximum of $0.4349^{\circ} .^{38}$

When subjected to FTIR scanning at wavelength range of $450-3500 \mathrm{~cm}^{-1}$ at room temperature, a broad absorption peak at $\sim 3320 \mathrm{~cm}^{-1}$ can be seen in Fig. 3, which arises due to $\mathrm{O}-\mathrm{H}$ stretching and the bending vibration of the intercalated free water molecules from to absorbed moisture. ${ }^{39}$ Absorption peaks centered at $\sim 2935$ and $\sim 2812 \mathrm{~cm}^{-1}$ correspond to the $\mathrm{C}-\mathrm{H}$ stretching due to the vibration of $-\mathrm{CH}_{3}$ and $-\mathrm{CH}_{2}$ groups in chitosan polymer. ${ }^{40}$ Absorption peaks near 1555, 1407, and $1027 \mathrm{~cm}^{-1}$ that ascribed to symmetric bending of $\mathrm{NH}_{2}, \mathrm{C}-\mathrm{N}$, and $\mathrm{C}-\mathrm{O}$ groups present in chitosan ${ }^{40-42}$ can also be observed in the figure. The presence of NiO nanoparticles in the thin film is

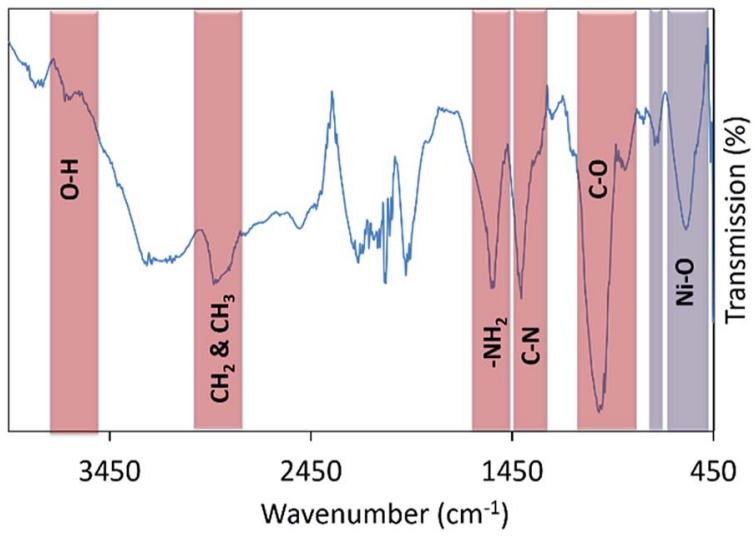

Fig. 3 FTIR spectrum of $\mathrm{NiO} /$ chitosan film. validated by the presence of two absorption peaks at 745 and $602 \mathrm{~cm}^{-1}$ which are assigned to the stretching vibrations of the $\mathrm{Ni}-\mathrm{O}$ bonds. ${ }^{43,44}$

The surface morphology of the NiO nanoparticles and elemental composition of the $\mathrm{NiO} /$ chitosan thin film are characterized using FESEM and EDX respectively with their results given in Fig. 3. As seen in Fig. 4(a), the FESEM image of the NiO nanoparticles at 130k magnification clearly shows a high yield of spherical nanoparticles with an average diameter of less than $25 \mathrm{~nm}$. Meanwhile, the presence of NiO nanoparticles and chitosan in the thin film is confirmed by element mapping, as shown in Fig. 4(b). Four main elements are observed, with the presence of carbon (C) and nitrogen (N) corresponding to the chitosan film while the nickel $(\mathrm{Ni})$ and oxygen $(\mathrm{O})$ elements correlate to the $\mathrm{NiO}$ nanoparticles. ${ }^{45,46}$ All the elements are well-defined with sharp contrasts. Further analysis carried out by EDX confirms that the nanohybrid comprises of the $\mathrm{C}, \mathrm{O}, \mathrm{N}$, and Ni elements, as given in Fig. 4(c). A Ni and $\mathrm{O}$ ratio of about $1.05: 0.95$ observed from the spectrum is consistent with the theoretical value of $\mathrm{NiO}$, while the presence of no other peaks in the spectrum indicates the purity of the fabricated $\mathrm{NiO} /$ chitosan film.
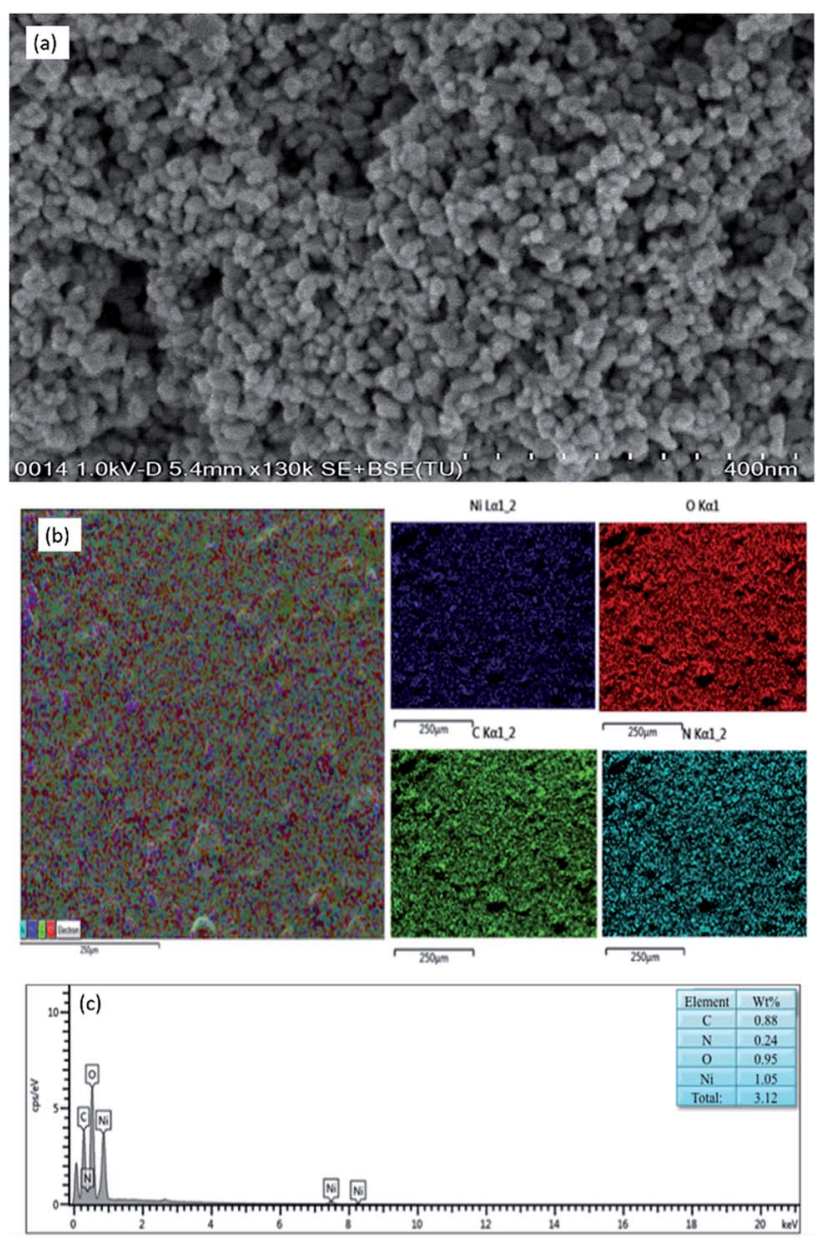

Fig. 4 (a) FESEM image of the NiO nanoparticles drop-casted on the ITO substrate, (b) EDX mapping of $\mathrm{Ni}, \mathrm{O}, \mathrm{C}$, and $\mathrm{N}$ elements, and (c) EDX analysis data of the plotted area. 
The size of the nanoparticles are not small enough to adversely affect the saturable absorption characteristics of the SA, leading to relatively typical SA behaviour. ${ }^{47}$ This was also demonstrated by Molli et al. ${ }^{48}$ who showed similar behaviour in vanadium pentoxide $\left(\mathrm{V}_{2} \mathrm{O}_{5}\right)$ nanoparticles.

The thermal stability of the prepared films is studied using a TGA4000 Perkin Elmer Pyris Diamond Thermogravimetric Analyzer. The results are recorded between temperature range of 30 to $900{ }^{\circ} \mathrm{C}$ under $\mathrm{N}_{2}$ atmosphere at a heating rate of $10{ }^{\circ} \mathrm{C} \mathrm{min}^{-1}$. The thermal stability of the $\mathrm{NiO} /$ chitosan film is depicted in Fig. 5, and from the TGA curves of the NiO/chitosan film, two thermal transitions at $28-259^{\circ} \mathrm{C}$ and $259-629^{\circ} \mathrm{C}$ can be observed. The initial weight loss of about $13.35 \%$ is observed within the temperature range of $28-259^{\circ} \mathrm{C}$ due to the removal of absorbed water and hydroxide molecules from the prepared film ${ }^{49}$ and also indicates the formation of stable NiO nanoparticles. ${ }^{50}$ The final thermal transitions occur within the temperature range of $259-629{ }^{\circ} \mathrm{C}$ with a weight reduction of about $52.54 \%$ might cause by the degradation of polysaccharide and decomposition of chitosan molecules. ${ }^{51}$ The weight loss reaches a plateau when the temperature is higher than $630{ }^{\circ} \mathrm{C}$ with a remaining weight of about $34.11 \%$. The total weight loss of the prepared films is found to be less as compared to other reports on chitosan, ${ }^{52}$ thus suggesting good thermal stability of the $\mathrm{NiO} /$ chitosan film.

The twin-detector measurement technique is used to measure the nonlinear absorption characteristics of the $\mathrm{NiO} /$ chitosan thin film. A mode-locked laser is used to generate a seed signal at a lasing wavelength of $1560 \mathrm{~nm}$ with a pulse width of $0.71 \mathrm{ps}$ and repetition rate of $28.17 \mathrm{kHz}$. The experimental data obtained, together with a fitted curve computed from the saturation model equation ${ }^{53}$ is given in Fig. 6. Based on the figure, the effective modulation depth, non-saturable losses, and saturation intensity of the $\mathrm{NiO} /$ chitosan thin film are calculated to be $\sim 11.50 \%, 88.50 \%$, and $4.34 \mathrm{~kW} \mathrm{~cm}{ }^{-2}$, respectively. The measured modulation depth the $\mathrm{NiO} /$ chitosan thin film is comparable to that of other materials such as $\mathrm{WS}_{2} /$ PVA at $2 \%,{ }^{54} \mathrm{Fe}_{3} \mathrm{O}_{4} / \mathrm{PVA}$ at $1.80 \%,{ }^{55}$ and gold nanorods/PVA at $4.06 \% .{ }^{56}$ These results indicated that the NiO/chitosan film has a significant potential for use as SA.

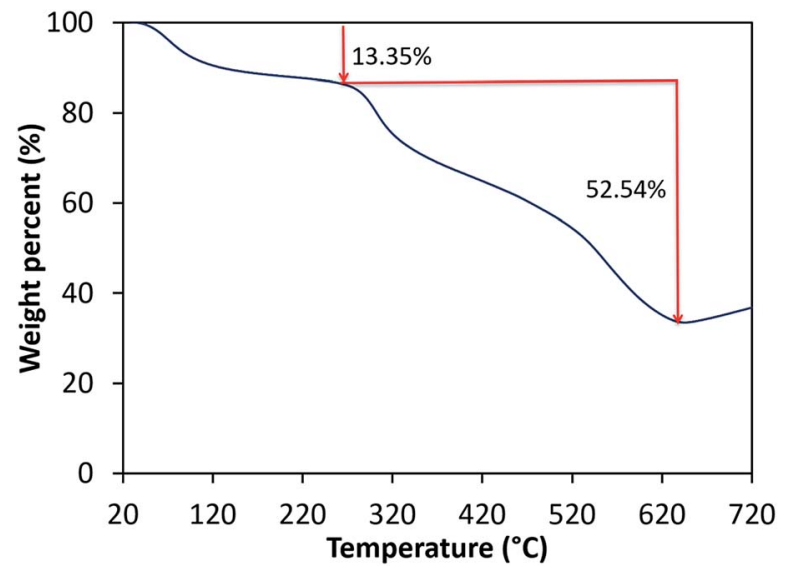

Fig. 5 The TGA plot of the prepared $\mathrm{NiO} /$ chitosan film.

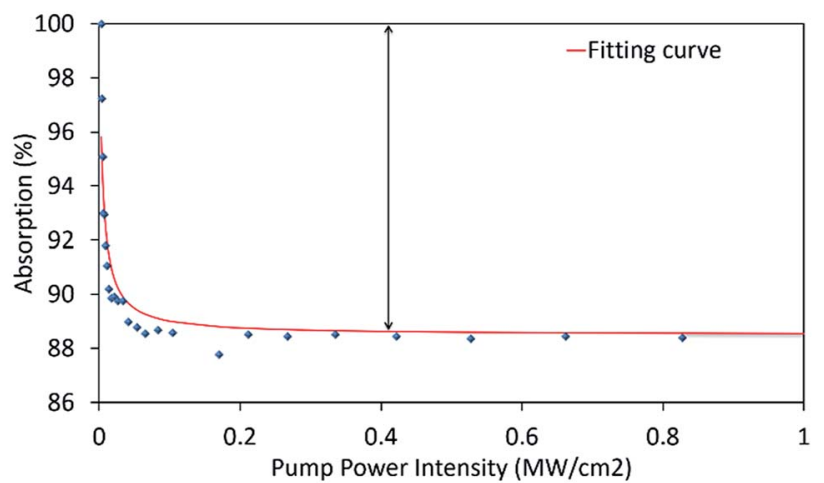

Fig. 6 The measured nonlinear absorption of the prepared NiO/chitosan SA.

\section{Q-switched optical fiber laser set-up}

Fig. 7 shows the experimental set-up of the proposed tunable passively Q-switched EDFL with the NiO/chitosan thin filmbased SA. A $980 \mathrm{~nm}$ laser diode (LD) acts as the pump sources and is connected to the $980 \mathrm{~nm}$ port of a $980 / 1550 \mathrm{~nm}$ wavelength division multiplexer (WDM). The common port of the WDM is now connected to a $0.89 \mathrm{~m}$ long erbium doped fiber (EDF) and is in turn connected to an isolator to ensure the unidirectional propagation of light in the cavity. The output of the isolator is connected to a fiber-based $80: 20$ optical coupler, which is used to extract approximately $20 \%$ of the propagating signal for analysis. The $80 \%$ port of the coupler on the other hand is connected to a tunable band pass filter (TBPF) with a tuning range up to $80 \mathrm{~nm}$, and subsequently to the SA and a polarization controller (PC). Finally, the PC is connected to the $1550 \mathrm{~nm}$ port of the WDM, thus completing the optical circuit. The laser cavity has a total length of approximately $9.1 \mathrm{~m}$ with its insertion loss measured to be $3.66 \mathrm{~dB}$. The $20 \%$ port of the optical circular is connected to another $50: 50$ optical coupler, with one end of the coupler connected to a Yokogawa AQ6370B optical spectrum analyzer (OSA) for optical spectrum and pulse shape measurements. The other end of the coupler is connected, in turn, to a Yokogawa DLM2054 mixed signal oscilloscope coupled with a photodetector, Anritsu MS2683 Radio-

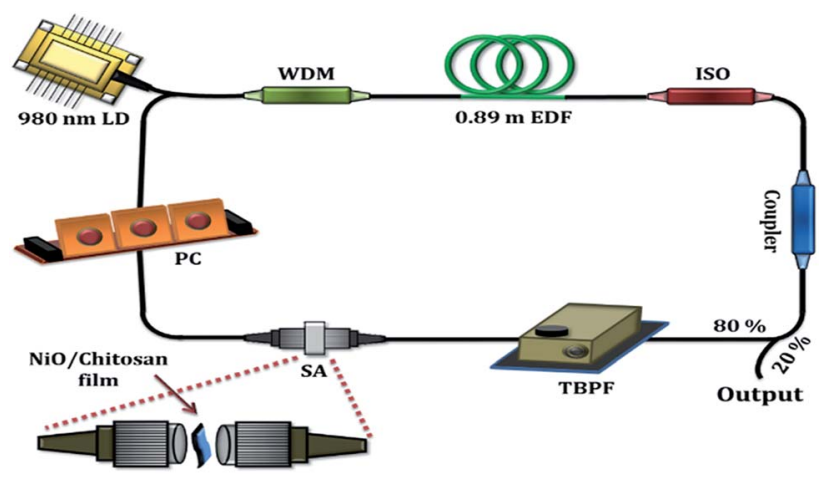

Fig. 7 Schematic illustration of the experimental set-up for the wavelength tunable passively Q-switched EDF laser cavity. 
Frequency Spectrum Analyzer (RFSA) and a Thorlabs power meter for the measurement of the output in the frequency domain, as well as the measurement of the signal to noise ratio (SNR) and the output power of output pulses.

\section{Results and discussions}

The CW lasing characteristics of the proposed laser are first determined by configuring the cavity in Fig. 7 with the omission of the SA. In this configuration, the lasing threshold of the proposed system under CW operation is determined to be 74.78 $\mathrm{mW}$, giving a laser output with a power of $0.13 \mathrm{~mW}$. Fig. 8 gives the optical spectrum of the CW signal taken from the system at a pump power of $171.30 \mathrm{~mW}$. As can be seen from the figure, the CW output has a lasing wavelength with a narrow peak centred at $1567.35 \mathrm{~nm}$, with a $3 \mathrm{~dB}$ bandwidth of $0.37 \mathrm{~nm}$.

Q-switched operation of the cavity is then achieved by integrating the $\mathrm{NiO} /$ chitosan based SA into the EDFL cavity, but without the TBPF present. Self-starting Q-switched operation at $1562 \mathrm{~nm}$ is observed at a threshold pump power of $104.90 \mathrm{~mW}$ with an average output power of $0.05 \mathrm{~mW}$. It is observed that when the $\mathrm{NiO} /$ chitosan based SA is removed from the cavity, no pulsed outputs are observed, indicating that the SA is responsible for the generation of pulses and not any other optical phenomena. Fig. 9 gives the oscilloscope trace of the Q-switched pulses for three different pump powers. It can be seen that the pulse trains becomes narrower as the pump power is increased from $140.70 \mathrm{~mW}$ to $244.50 \mathrm{~mW}$, along with the interval between pulses decreasing gradually as well. This is expected of the pulsed output and is a typical feature of passive Q-switching operation. It can also be seen that the Q-switched pulse train has uniform pulse shapes at different pump powers, indicating a high level of stability.

The typical output characteristics of the Q-switched laser at a pump power of $171.30 \mathrm{~mW}$ is given in Fig. 10. Fig. 10(a) shows the laser emission spectra with lasing observed at $1562 \mathrm{~nm}$ with a $3 \mathrm{~dB}$ bandwidth of $1.33 \mathrm{~nm}$. It should also be noted that compared to CW lasing, the spectrum obtained under Qswitched lasing is broadened, with a shift to towards the low wavelength region. The broadening of the lasing spectrum is caused by the self-phase modulation (SPM) effect of the signal

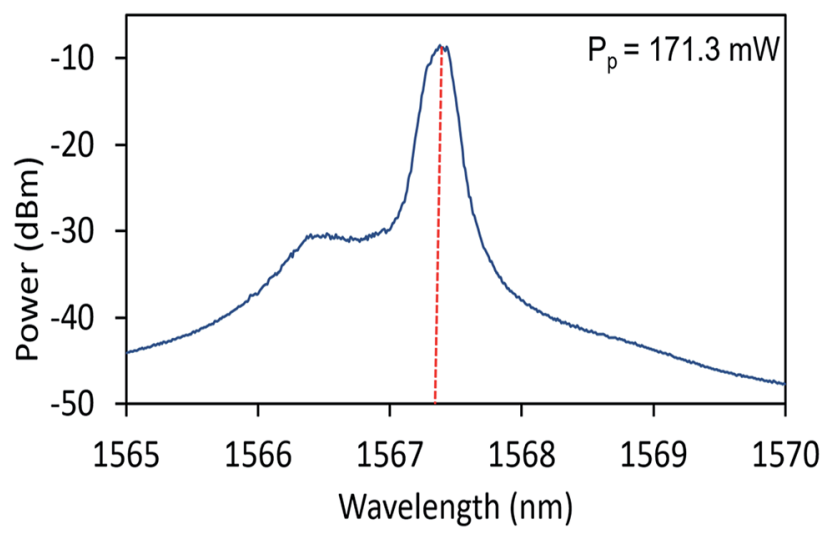

Fig. 8 Optical spectrum of CW lasing output.

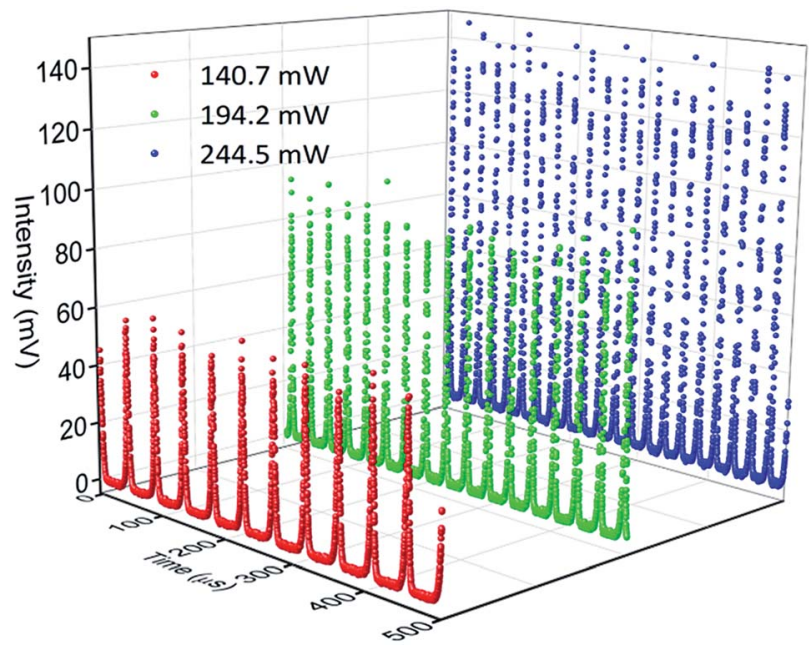

Fig. 9 Q-switched pulse trains at pump powers of $140.7 \mathrm{~mW}, 194.2$ $\mathrm{mW}$ and $244.5 \mathrm{~mW}$.

lasing in the cavity, as well as the shift of the system towards a higher gain region to compensate for losses induced by the insertion of the SA into the cavity. ${ }^{57}$ This provides additional validation that the $\mathrm{NiO} /$ chitosan based $\mathrm{SA}$ is responsible for inducing the Q-switched pulses in the cavity. As seen in Fig. 10(b), the laser cavity produces a stable pulse train with a repetition rate of $29.30 \mathrm{kHz}$ and time interval between adjacent pulses of $34 \mu \mathrm{s}$. The focused view of the typical single pulse profile obtained under the afore-mentioned conditions is given in Fig. 10(c). It can be seen that the pulse envelope possess a symmetrical Gaussian-like shape with a full width at half maximum (FWHM) value of $2.90 \mu \mathrm{s}$, comparable to that reported works. ${ }^{58,59}$ From the radio frequency (RF) spectrum presented in Fig. 10(d), the SNR value is measured to be $\sim 43.26 \mathrm{~dB}$ at a fundamental frequency of $29.30 \mathrm{kHz}$, which demonstrates the good Q-switched stability. The measured SNR value is comparable to that obtained from similarly reported systems. ${ }^{\mathbf{6 0 , 6 1}}$

The stability of the laser system is observed by monitoring its power stability over a period of one hour. The RF spectra is recorded at intervals of every 10 minutes at a fixed pump power and repetition rates of $171.30 \mathrm{~mW}$ and $29.30 \mathrm{kHz}$, with the 3D plot of the RF spectra given in Fig. 11(a). No obvious changes are observed in the fundamental frequency and RF intensity during the test period, thus demonstrating that the generated pulses are highly stable. In addition to this, the trend of the SNR values as in Fig. 11(b), also indicates a highly stable pulsed output, with changes in the SNR value and fundamental frequency shows of less than $1 \mathrm{dBm}$ and $0.10 \mathrm{kHz}$, respectively during the same observation period.

The response of the pulse duration and repetition rate of the Q-switched pulses against the pump power is given in Fig. 12(a). From the figure, it can be observed that the pulse duration and repetition rate increases linearly and decreases somewhat exponentially over a pump power range of 104.90 to $244.50 \mathrm{~mW}$. The repetition rate at the minimum pump power is approximately $14.49 \mathrm{kHz}$, and increased to $42.66 \mathrm{kHz}$ at the maximum 

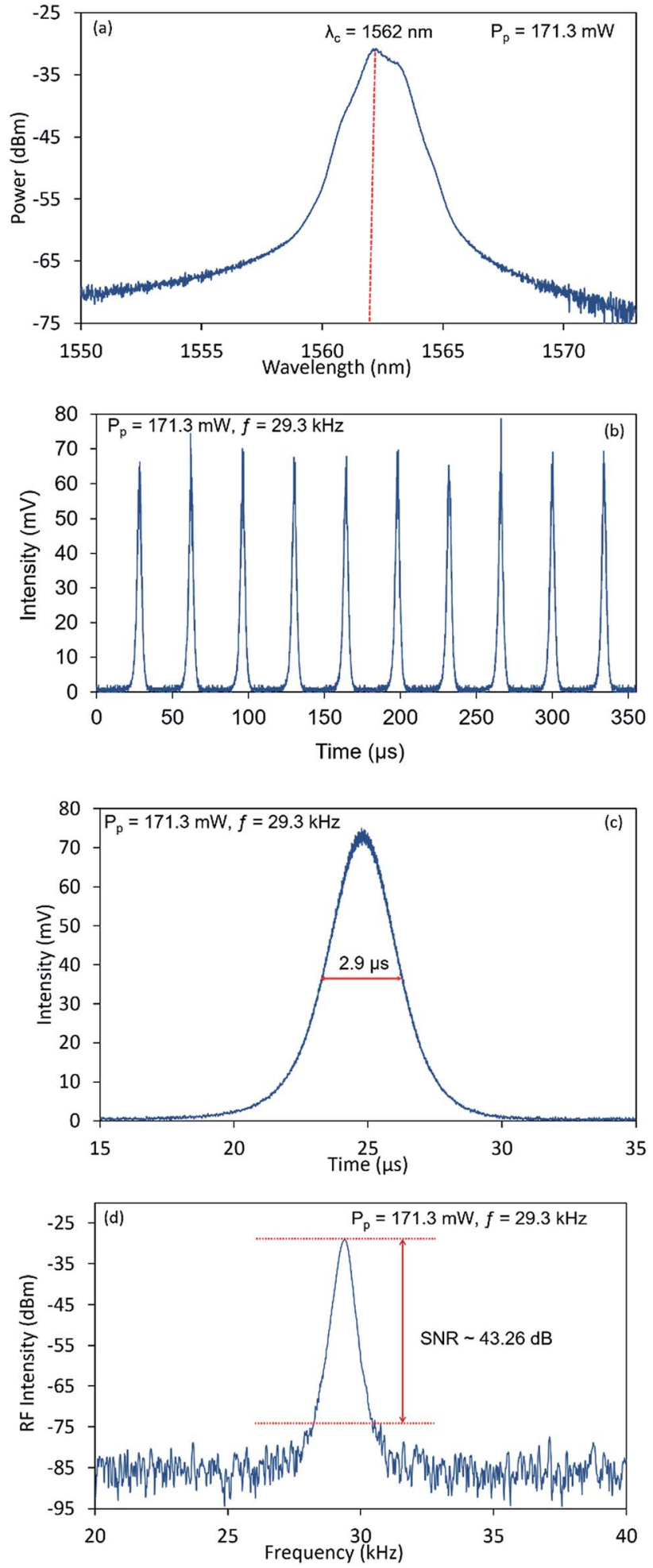

Fig. 10 Typical characteristics of the Q-switched pulses generated from the EDF laser at a pump power of $171.30 \mathrm{~mW}$, showing (a) the optical spectrum output, (b) the oscilloscope trace of the pulse train, (c) a single pulse profile and (d) the RF spectrum at fundamental frequency, $f_{\mathrm{o}}=29.30 \mathrm{kHz}$.

pump power. The pulse width on the other hand is approximately $8.50 \mu \mathrm{s}$ at the minimum pump power, and decreases exponentially until it reaches a value of $3.40 \mu$ s at a pump power
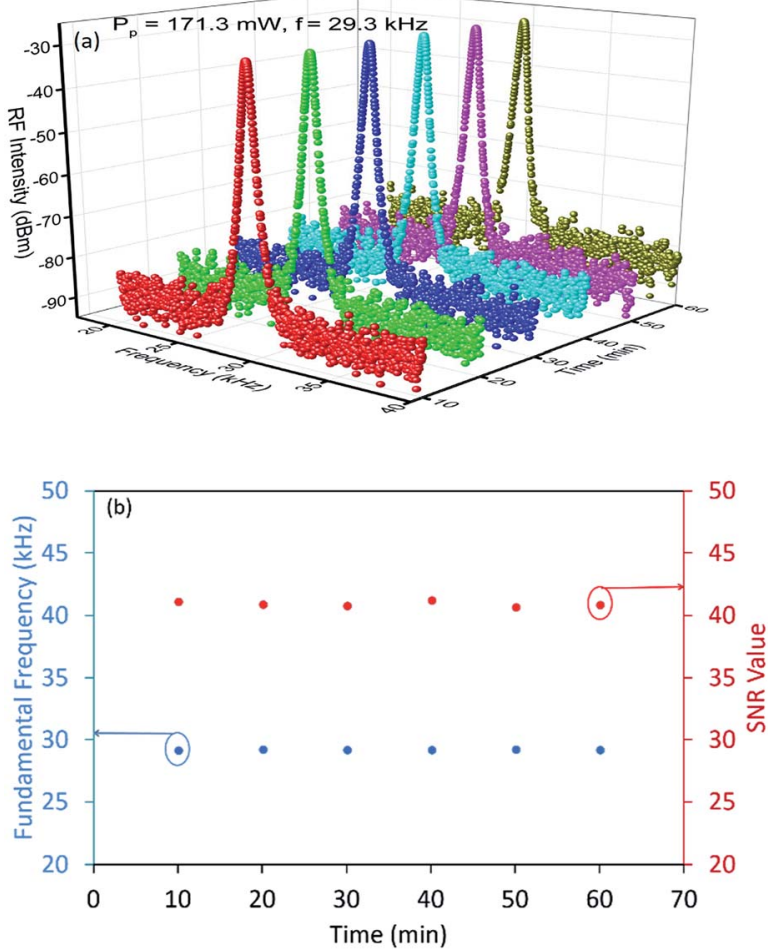

Fig. 11 (a) 3D plot of the RF spectra and (b) fundamental frequency and SNR value against time, recorded at 10 minutes intervals over a period of 1 hour.
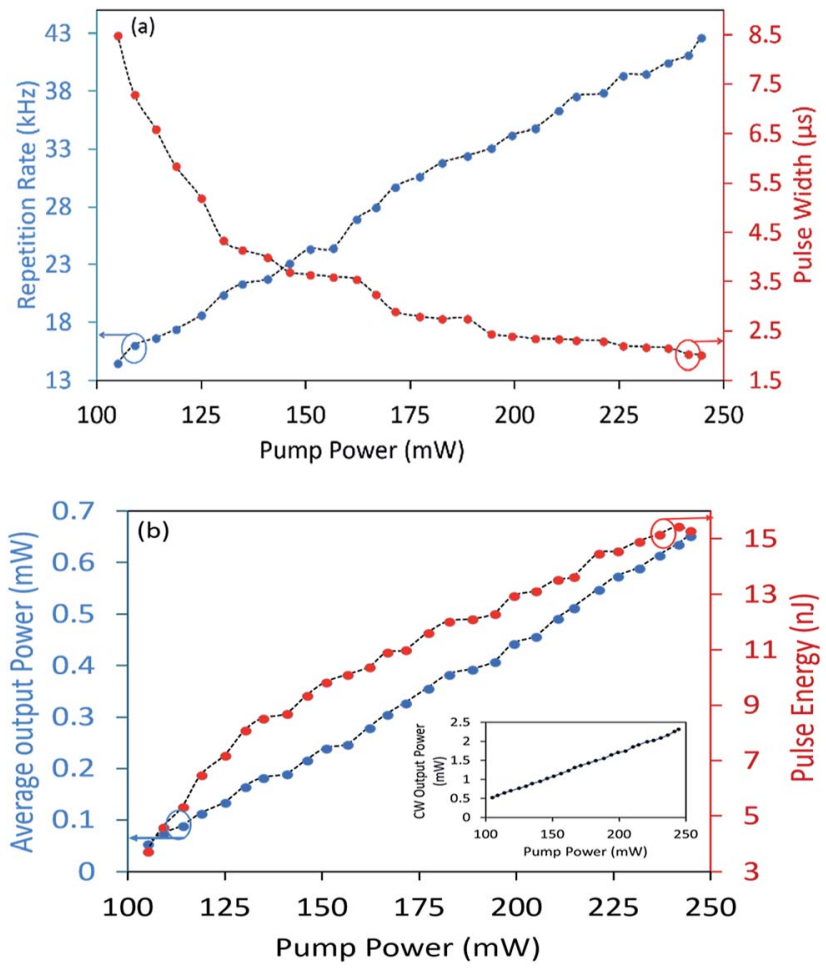

Fig. 12 (a) Pulse width and repetition rate, and (b) pulse energy and average output power of the pulsed output against the pump power. Inset: The CW output power versus the pump power. 

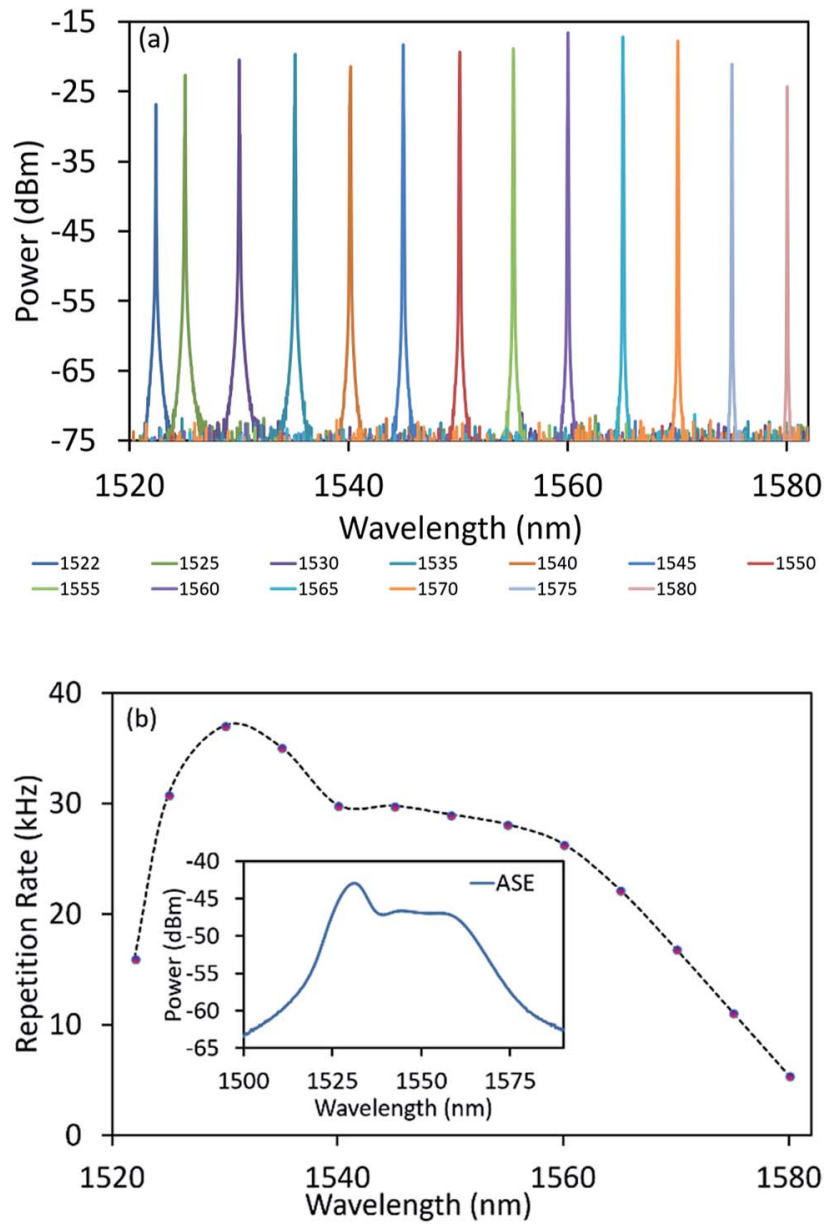

Fig. 13 (a) The optical spectra of the $Q$-switching operation from $1522 \mathrm{~nm}$ to $1580 \mathrm{~nm}$ and (b) the repetition rate as a function of the tunable lasing wavelength. The inset of the figure shows the ASE spectrum of the EDF when pumped at $194.20 \mathrm{~mW}$.

of $194.20 \mathrm{~mW}$, where it now decreases at a slower rate until reaching $2.02 \mu \mathrm{s}$ at the maximum pump power.

Fig. 12(b) gives the response of the average output power and pulse energy against the rising pump power, and it can be seen from the figure that both the average output power and pulse energy grows linearly against the pump power, reaching its maximum values of $0.65 \mathrm{~mW}$ and $15.30 \mathrm{~nJ}$ at the maximum pump power. The dependence of the average output power of $\mathrm{CW}$ to the pump power is depicted in the inset of Fig. 12(b). From the figure, it can be seen that the average output power increases linearly with the incident pump power until a maximum output power of 2.32 $\mathrm{mW}$ is reached at a pump power of $244.50 \mathrm{~mW}$. The slightly low laser slope efficiency is due to the insertion loss of the $\mathrm{NiO} /$ chitosan SA. The pulse energy obtained in this work is comparable to that reported in other Q-switched fiber lasers using graphene oxide ${ }^{62}$ and zinc oxide ( $\left.\mathrm{ZnO}\right) .{ }^{63}$ Moreover, the same trends can be observed should the pump power be reduced, indicating that the SA has not sustained any thermal damage and that its damage threshold is most likely higher than the maximum pump power available in this work.

At this point, the TBPF is introduced into the laser cavity to enable wavelength tunability of the proposed laser. The resulting tunability spectrum is given in Fig. 13, and shows a stable output over a wavelength range stretching from 1522 to $1580 \mathrm{~nm}$. As shown in Fig. 13(a), a tuning range of $58 \mathrm{~nm}$ can be obtained covering the $\mathrm{C}$ and $\mathrm{L}$ bands as well as portions of the $\mathrm{S}$ band. The dependence of the repetition rate of the pulsed output at different tuning wavelengths is given in Fig. 13(b), and shows that the repetition rate increased from $16 \mathrm{kHz}$ to 37.04 $\mathrm{kHz}$ when the wavelength is tuned from $1522 \mathrm{~nm}$ to $1530 \mathrm{~nm}$. Increasing the lasing wavelength further results in the repetition rate decreasing to $5.38 \mathrm{kHz}$ at a wavelength of $1580 \mathrm{~nm}$. The observed trend of the repetition rate at different lasing wavelengths is observed to be very similar to that of the amplified spontaneous emission (ASE) spectrum of the EDF when pumped at $194.20 \mathrm{~mW}$, as seen in the inset of Fig. 13(b). This is attributed to the fast population inversion from the strong intracavity laser at the larger gain region of the EDF, which in turn makes the bleaching process of the NiO/chitosan SA faster and thus resulting in a higher larger repetition rate ${ }^{64}$ Furthermore, the dependency of the tunability on the bandwidth of the EDF gain medium shows no noticeable Q-switched pulses beyond the tunable wavelength range.

Table 1 Comparison of the performance of various SA in C-band region Q-switched fiber laser using EDF as gain medium ${ }^{a}$

\begin{tabular}{|c|c|c|c|c|c|c|}
\hline BP & 1562.35 & & $9.61-44.72$ & 9.80 & 81.50 & 65 \\
\hline ZnNPs & 1565.50 & - & $12.30-43$ & - & 2.60 & 63 \\
\hline $\mathrm{Fe}_{3} \mathrm{O}_{4} / \mathrm{PVA}$ & 1557 & - & $8.50-28$ & 6 & 71 & 24 \\
\hline $\mathrm{Fe}_{3} \mathrm{O}_{4} / \mathrm{PI}$ & 1562 & - & $5.50-49$ & 3.50 & - & \\
\hline Ag/MTMS & $1552.90-1580.20$ & 27.30 & $10.50-24.40$ & 6.54 & - & 68 \\
\hline $\mathrm{MoS}_{2} / \mathrm{PVA}$ & $1519.60-1567.70$ & 48.10 & $8.77-43.47$ & 3.30 & 160 & 69 \\
\hline $\mathrm{NiO} /$ Chitosan & $1522-1580$ & 58 & $14.49-42.66$ & 2.02 & 15.30 & This work \\
\hline
\end{tabular}

${ }^{a} \mathrm{BP}=$ Black phosphorus; $\mathrm{GO}=$ graphene oxide; $\mathrm{ZnNPs}=$ zinc nanoparticles; $\mathrm{Fe}_{2} \mathrm{O}_{4}=$ ferroferric oxide nanoparticles; PI $=$ polyimide; CuNP $=$ copper nanoparticles; MWCNT $=$ multiwall carbon nanotubes; $\mathrm{Ag}=$ silver nanoparticles; $\mathrm{MTMS}^{=}$methyltrimethoxysilane; $_{\mathrm{MoS}}=$ molybdenum disulfide; PVA = polyvinyl alcohol. 
Table 1 provides a comparison of the output performance parameters for the proposed Q-switched laser against similar designs utilizing various materials as SAs.

It can be seen that the proposed system of this work is able to cover a wide C-band region as compared to other works. The tunability range of the proposed laser is also significantly better, as shown in Table 1. It is noted that while other works ${ }^{67,68}$ are able to be extended into the S-band region, similar to that of this work, the C-band region covered is not as broad. Furthermore, while other works successfully generate Q-switched pulses with a larger maximum pulse energy, ${ }^{24,65,67,69}$ the pulses produced in this work have the advantage of a shorter minimum pulse width. The system proposed by Mansoor et al. ${ }^{62}$ has a comparable minimum pulse width, though these pulses have a lower maximum pulse energy as compared that obtained in this work. As reported by T. Y. Tsai et al. ${ }^{70}$ output pulses from Q-switched operations with a shorter pulse width is highly preferred for photonics applications. Thus, the proposed work has significant substantial potential for the generation of passively Qswitched pulses in the wideband C-band region for various photonics applications.

The maximum average output power can also be optimized by using a different optical coupler ratio, such as $90: 10$ or $95: 5$ to increase the power of the signal propagating in the cavity ${ }^{71}$ and also by reducing the cavity length. ${ }^{72}$ Furthermore, the careful control of cavity losses ${ }^{73}$ as well as the use of fiber Bragg gratings to clamp the lasing wavelength ${ }^{74}$ can also boost the maximum average power output. Therefore, the proposed work with the generated pulses has substantial potential for the passively Q-switched pulses in the wideband C-band region especially in the photonics applications.

\section{Conclusion}

In this work, a broad range wavelength tunable passively Qswitched EDF laser with a $\mathrm{NiO} /$ chitosan thin film based SA is proposed and demonstrated. The proposed laser shows the feasibility of the $\mathrm{NiO} /$ chitosan thin film as an $\mathrm{SA}$, and is capable of generating a tuning output over a range of $58 \mathrm{~nm}$ from 1522 to $1580 \mathrm{~nm}$, covering the $\mathrm{C}$ and $\mathrm{L}$ bands and elements of the $\mathrm{S}$ band as well. A stable pulse train is obtained at a low threshold power of $104.90 \mathrm{~mW}$ at $1562 \mathrm{~nm}$, with the highest pulse energy of $15.30 \mathrm{~nJ}$ and maximum repetition rate of $42.66 \mathrm{kHz}$ obtained at the maximum pump power. A minimum pulse width of 2.02 $\mu \mathrm{s}$ is also obtained at this pump power. The output is highly stable, with an SNR of around $43 \mathrm{~dB}$. The advantages of the chitosan film, which include its biocompatibility, non-toxicity and high thermal resistance make it a highly attractive candidate material for SA fabrication. The proposed SA will have many practical photonics applications, particularly for optical signal processing and optical fiber communication systems.

\section{Conflicts of interest}

There are no conflicts to declare.

\section{Acknowledgements}

This research was funded by the grant LRGS (2015) NGOD/UM/ KPT from the Ministry of Higher Education, Malaysia and also the grants RU 001 - 2017 and RP 029B - 15 AFR from the University of Malaya. We would also like to acknowledge the Distinguished Visiting Fellowship for H. Ahmad from the Royal Academy of Engineering (UK) to City, University of London.

\section{References}

1 A. F. El-Sherif and T. A. King, Opt. Lett., 2003, 28, 22-24.

2 C. Cuadrado-Laborde, A. Díez, J. L. Cruz and M. V. Andrés, Opt. Lett., 2009, 34, 2709-2711.

3 Y. Lu and X. Gu, IEEE Photonics J., 2014, 6, 1-7.

4 Z. Yu, M. Malmström, O. Tarasenko, W. Margulis and F. Laurell, Opt. Express, 2010, 18, 11052-11057.

5 J. Ren, S. Wang, Z. Cheng, H. Yu, H. Zhang, Y. Chen, L. Mei and P. Wang, Opt. Express, 2015, 23, 5607-5613.

6 O. Buryy, A. Iznin, I. Syvorotka, D. Sugak, S. Ubizskii and M. Vakiv, Acta Phys. Pol., A, 2010, 117, 238-243.

7 O. L. Antipov, N. G. Zakharov, M. Fedorov, N. M. Shakhova, N. N. Prodanets, L. B. Snopova, V. V. Sharkov and R. Sroka, Med Laser Appl, 2011, 26, 67-75.

8 S. J. Ahn, J. H. Kim, D. W. Kim, H. S. Kim, Y. C. Kim, S. Ahn and C. Kim, Phys. Status Solidi B, 2004, 241, 1650-1653.

9 T. S. Jang, S. S. Lee and Y. G. Kim, Ultrasonics, 2004, 42, 837841.

10 N. Trela, P. R. Kaczmarek and K. M. Abramski, Transparent Optical Networks, 2007. ICTON'07. 9th International Conference on IEEE, 2007, vol. 4, pp. 244-247.

11 S. R. Yemineni, A. Arokiaswami and P. Shum, Lasers and Electro-Optics Pacific Rim (CLEO-PR), 2017 Conference on IEEE, 2017, pp. 1-12.

12 B. Chen, X. Zhang, K. Wu, H. Wang, J. Wang and J. Chen, Opt. Express, 2015, 23, 26723-26737.

13 Z. Luo, Y. Huang, J. Weng, H. Cheng, Z. Lin, B. Xu, Z. Cai and H. Xu, Opt. Express, 2013, 21, 29516-29522.

14 D. Wu, Z. Cai, Y. Zhong, J. Peng, Y. Cheng, J. Weng, Z. Luo and H. Xu, IEEE J. Sel. Top. Quantum Electron., 2017, 23, 7-12.

15 M. F. M. Rusdi, A. A. Latiff, M. C. Paul, S. Das, A. Dhar, H. Ahmad and S. W. Harun, Opt. Laser Technol., 2017, 89, 16-20.

16 Z. Qin, G. Xie, C. Zhao, S. Wen, P. Yuan and L. Qian, Opt. Lett., 2016, 41, 56-59.

17 B. Xu, Y. Wang, J. Peng, Z. Luo, H. Xu, Z. Cai and J. Weng, Opt. Express, 2015, 23, 7674-7680.

18 B. Xu, Y. Cheng, Y. Wang, Y. Huang, J. Peng, Z. Luo, H. Xu, Z. Cai, J. Weng and R. Moncorgé, Opt. Express, 2014, 22, 28934-28940.

19 A. Nady, M. Ahmed, A. Numan, S. Ramesh, A. Latiff, C. Ooi, H. Arof and S. Harun, J. Mod. Opt., 2017, 64, 1315-1320.

20 D. Mohammed, W. A. Khaleel and A. Al-Janabi, Opt. Laser Technol., 2017, 97, 106-110.

21 A. Nady, A. Latiff, A. Numan, C. R. Ooi and S. Harun, Laser Phys., 2018, 28, 085106. 
22 B. Xuekun, M. Chengbo, X. Luxi, W. Shaofei, P. Shengli and Z. Xianglong, Appl. Phys. Express, 2016, 9, 042701.

23 J. Guo, H. Zhang, C. Zhang, Z. Li, Y. Sheng, C. Li, X. Bao, B. Man, Y. Jiao and S. Jiang, Opt. Mater. Express, 2017, 7, 3494-3502.

24 D. Mao, X. Cui, W. Zhang, M. Li, T. Feng, B. Du, H. Lu and J. Zhao, Photonics Res., 2017, 5, 52-56.

25 H. Ahmad, A. S. Sharbirin, A. Muhamad, M. Z. Samion, S. A. Reduan, A. Z. Zulkifli and M. F. Ismail, J. Lightwave Technol., 2017, 35, 2470-2475.

26 N. Aziz, A. Latiff, M. Lokman, E. Hanafi and S. Harun, Chin. Phys. Lett., 2017, 34, 044202.

27 H. Ahmad, C. S. J. Lee, M. A. Ismail, Z. A. Ali, S. A. Reduan, N. E. Ruslan, M. F. Ismail and S. W. Harun, Opt. Commun., 2016, 381, 72-76.

28 A. Nady, M. H. M. Ahmed, A. A. Latiff, A. Numan, C. H. R. Ooi and S. W. Harun, Laser Phys., 2017, 27, 065105.

29 M. Deshpande, K. N. Patel, V. P. Gujarati, K. Patel and S. Chaki, Adv. Mater. Res., 2016, 1141, 65.

30 H.-T. Wang, D. K. Mishra, P. Chen and J.-M. Ting, J. Alloys Compd., 2014, 584, 142-147.

31 R. S. Kate, S. A. Khalate and R. J. Deokate, J. Alloys Compd., 2018, 734, 89-111.

32 N. Duraisamy, A. Numan, S. O. Fatin, K. Ramesh and S. Ramesh, J. Colloid Interface Sci., 2016, 471, 136-144.

33 D. Homa, Q. Zhang, C. Hill, G. Kaur and G. Pickrell, Curr. Appl. Phys., 2014, 14, 1396-1400.

34 N. Sattarahmady, H. Heli and R. D. Vais, Biosens. Bioelectron., 2013, 48, 197-202.

35 L. H. Chen, T. Li, C. C. Chan, R. Menon, P. Balamurali, M. Shaillender, B. Neu, X. M. Ang, P. Zu, W. C. Wong and K. C. Leong, Sens. Actuators, B, 2012, 169, 167-172.

36 R. Raghunandhan, L. H. Chen, H. Y. Long, L. L. Leam, P. L. So, X. Ning and C. C. Chan, Sens. Actuators, B, 2016, 233, 31-38.

37 S. Rakshit, S. Chall, S. S. Mati, A. Roychowdhury, S. Moulik and S. C. Bhattacharya, RSC Adv., 2013, 3, 6106-6116.

38 B. Ingham and M. F. Toney, in Metallic Films for Electronic, Optical and Magnetic Applications, Woodhead Publishing, 2014, pp. 3-38, DOI: 10.1533/9780857096296.1.3.

39 M. El-Kemary, N. Nagy and I. El-Mehasseb, Mater. Sci. Semicond. Process., 2013, 16, 1747-1752.

40 M. AbdElhady, Int. J. Carbohydr. Chem., 2012, 2012, 1-6.

41 L. Vitali, K. C. Justi, M. Laranjeira and V. T. Fávere, Polimeros, 2006, 16, 116-122.

42 Y. Wang, A. Pitto-Barry, A. Habtemariam, I. RomeroCanelon, P. J. Sadler and N. P. Barry, Inorg. Chem. Front., 2016, 3, 1058-1064.

43 P. Vijayadarshan, T. Mohan, J. V. Viswanath, K. Venugopal, N. S. Rao and A. Gupta, Mater. Sci. Res. India, 2017, 14, 37-42.

44 T. Shahid, T. Khan, M. Zakria, R. Shakoor and M. Arfan, J. Mater. Sci. Eng., 2016, 5, 6.

45 J. Safari and L. Javadian, RSC Adv., 2014, 4, 48973-48979.

46 Y. Chuminjak, S. Daothong, P. Reanpang, J. P. Mensing, D. Phokharatkul, J. Jakmunee, A. Wisitsoraat, A. Tuantranont and P. Singjai, RSC Adv., 2015, 5, 6779567802 .
47 J. K. Anthony, H. C. Kim, H. W. Lee, S. K. Mahapatra, H. M. Lee, C. K. Kim, K. Kim, H. Lim and F. Rotermund, Opt. Express, 2008, 15, 11193-11202.

48 M. Molli, G. D. Salian, S. K. Aditha, S. V. Muthukumar, T. M. Rattan, S. Amrithapandian, B. K. Panigrahi and V. Kamisetti, AIP Conf. Proc., 2012, 233-236.

49 A. Ashok, A. Kumar, R. R. Bhosale, M. A. H. Saleh and L. J. Van den Broeke, RSC Adv., 2015, 5, 28703-28712.

50 T. Marimuthu, S. Mohamad and Y. Alias, Synth. Met., 2015, 207, 35-41.

51 B. Lim, C. Poh, C. Voon and H. Salmah, Appl. Mech. Mater., 2015, 754-755, 34-38.

52 M. Karpuraranjith and S. Thambidurai, Int. J. Biol. Macromol., 2017, 104, 1753-1761.

53 A. Martinez and S. Yamashita, in Carbon Nanotubes Applications on Electron Devices, InTech, 2011.

54 L. Li, Y. Wang, Z. F. Wang, X. Wang and G. Yang, Opt. Commun., 2018, 406, 80-84.

55 J. Koo, J. Lee, J. Kim and J. H. Lee, J. Lumin., 2018, 195, 181186.

56 X.-D. Wang, Z.-C. Luo, H. Liu, N. Zhao, M. Liu, Y.-F. Zhu, J.-P. Xue, A.-P. Luo and W.-C. Xu, Opt. Commun., 2015, 346, 21-25.

57 H. Ahmad, S. Ooi, Z. Tiu and B. Rahman, Opt. Commun., 2018, 426, 1-8.

58 M. Zhang, G. Hu, G. Hu, R. C. T. Howe, L. Chen, Z. Zheng and T. Hasan, Sci. Rep., 2015, 5, 17482.

59 Y. Chen, G. Jiang, S. Chen, Z. Guo, X. Yu, C. Zhao, H. Zhang, Q. Bao, S. Wen and D. Tang, Opt. Express, 2015, 23, 1282312833.

60 L. Baole, C. Haowei, J. Man, C. Xiaoming, R. Zhaoyu and B. Jintao, Laser Phys., 2013, 23, 045111.

61 Y. Chen, C. Zhao, S. Chen, J. Du, P. Tang, G. Jiang, H. Zhang, S. Wen and D. Tang, IEEE J. Sel. Top. Quantum Electron., 2014, 20, 315-322.

62 A. Mansoor, B. A. Hamida, T. Eltaif, E. Ismail, N. Kadir, S. Khan and S. Harun, J. Opt. Commun., 2018, 307-310.

63 P. Zaca-Morán, J. G. Ortega-Mendoza, G. J. Lozano-Perera, L. C. Gómez-Pavón, G. F. Pérez-Sánchez, J. P. PadillaMartínez and C. Felipe, Laser Phys., 2017, 27, 105101.

64 Y. Huang, Z. Luo, Y. Li, M. Zhong, B. Xu, K. Che, H. Xu, Z. Cai, J. Peng and J. Weng, Opt. Express, 2014, 22, 2525825266.

65 N. N. Razak, A. A. Latiff, Z. Zakaria and S. W. Harun, Photonics Lett. Pol., 2017, 9, 72-74.

66 A. Muhammad, M. Ahmad, R. Zakaria, H. Rahim, S. Yusoff, K. Hamdan, H. Yusof, H. Arof and S. Harun, Chin. Phys. Lett., 2017, 34, 034205.

67 H. Ahmad, S. N. M. Hassan, F. Ahmad, M. Z. Zulkifli and S. W. Harun, Opt. Commun., 2016, 365, 54-60.

68 H. Ahmad, N. E. Ruslan, Z. A. Ali, S. A. Reduan, C. S. J. Lee, R. A. Shaharuddin, N. Nayan and M. A. Ismail, Opt. Commun., 2016, 381, 85-90.

69 Y. Huang, Z. Luo, Y. Li, M. Zhong, B. Xu, K. Che, H. Xu, Z. Cai, J. Peng and J. Weng, Opt. Express, 2014, 22, 2525825266.

70 T.-Y. Tsai and Y.-C. Fang, Opt. Express, 2009, 17, 1429-1434. 
71 W. Liu, X. Hu, L. Ying, S. Chen, J. Zhang, H. Akiyama, Z. Cai and B. Zhang, Sci. Rep., 2015, 5, 9600.

72 N. A. M. A. Hambali, M. A. Mahdi, M. H. Al-Mansoori, M. I. Saripan and A. F. Abas, Appl. Opt., 2009, 48, 5055-5060.
73 H. Ahmad, M. Z. Zulkifli, A. A. Latif and S. W. Harun, Opt. Commun., 2009, 24, 4771-4775.

74 S. W. Harun, S. K. Low, P. Poopalan and H. Ahmad, IEEE Photonics Technol. Lett., 2002, 14, 293-295. 